\title{
Dynamics of Lipase Catalyzed Enzymatic Degradation of Poly(bisphenol-A carbonate)
}

\author{
G. Sivalingam, Giridhar Madras \\ Department of Chemical Engineering, Indian Institute of Science, Bangalore-12, India
}

Received 19 February 2003; accepted 21 May 2003

\begin{abstract}
Poly(bisphenol-A carbonate) (PC) was degraded in solution at various temperatures $\left(26-70^{\circ} \mathrm{C}\right)$ by different lipases, Candida Rugosa (CR), Hog Pancreas (HP), Lipolase (LL), and Novozyme (NV), in various solvents. The degradation of PC was monitored using gel permeation chromatography (GPC). The molecular weights of oligomers obtained were around $\sim 1,400$ irrespective of the lipases used. Continuous distribution kinetics was proposed to determine the rate coefficients of the polymers and deactivation of the enzyme. The FTIR analysis of the polymer before and after degradation showed that there is large reduction of carbonate linkages and generation of hydroxyl and acidic
\end{abstract}

groups in the broken chains. The optimal temperatures for $\mathrm{HP}$ and other lipases were 50 and $60^{\circ} \mathrm{C}$, respectively. HP showed higher degradation activity at lower temperatures and the overall degradability of the lipases was in the order of LL $>$ CR $>N V>$ HP. The effect of viscosity and polarity of the solvents on the degradation of the polycarbonate was also investigated. While the degradation rate decreased with viscosity, it increased with polarity for the solvents. (c) 2003 Wiley Periodicals, Inc. J Appl Polym Sci 91: 2391-2396, 2004

Key words: enzymes; degradation; polycarbonate; continuous distribution kinetics; lipases; solvent effect

\section{INTRODUCTION}

Biodegradable polymers provide an attractive alternative to conventional nonbiodegradable polymers, both from environmental and biomedical applications. Enzymes are effective in a wide variety of commercial reactions, such as hydrolysis, transesterification, and acylation. ${ }^{1}$ Some enzymes, such as lipases, are stable and active in nearly water-immiscible organic solvents. ${ }^{2,3}$ It is speculated that a thin layer of water remains tightly bound to the enzyme. This water acts as a protective sheath along the enzyme's hydrophilic surfaces and allows retention of its native confirmation and hence activity, even in abiotic solvents. ${ }^{4}$ These desirable properties of lipases make them versatile in biodegradation of polymers in organic solvents.

Poly(bisphenol-A carbonate) is currently of great commercial importance as high-performance plastic, such as bulletproof and eye glasses, sheathings, and computer disks. It is of interest to examine the biodegradability of such polymers from their end application and ecological viewpoints. There are reports on the biodegradation of aliphatic polyesters by lipases ${ }^{5,6}$ and by microorganisms in soil. ${ }^{7}$ Polycarbonates with aliphatic components are also degradable by microorganisms. ${ }^{8,9}$ It has also been reported that the polycar-

Correspondence to: G. Madras (giridhar@chemeng.iisc. ernet.in).

Journal of Applied Polymer Science, Vol. 91, 2391-2396 (2004) (C) 2003 Wiley Periodicals, Inc. bonates with side chains connected by a polycarbonate are degraded by lipases and the hydrolysis of the main chain has remained questionable. ${ }^{4}$

Recently Sivalingam et al. ${ }^{10}$ showed that the enzyme activity on biodegradation of polymers is mainly dependent on the solvent properties and showed that the enzyme activity increases with polarity and decreases with viscosity of the solvent. The organic species such as nucleophiles are reported to alter the substrate specificity and improve the stability of the enzymes. ${ }^{11}$ The products obtained by biodegradation of aliphatic polycarbonate are $\mathrm{CO}_{2}$ and acids. ${ }^{9}$ For degradation of polymers in solution, the solubility of polymer in organic solvent is crucial.

In the present study, the biodegradation kinetics of poly(bisphenol-A carbonate), optimal temperatures, and the influence of solvent with different lipases were investigated. Continuous distribution kinetics models were developed taking the enzyme deactivation into account and the rate coefficients and enzyme deactivation coefficients were determined.

\section{Experimental section}

Materials

Poly(bisphenol-A carbonate) [average molecular weight 64,000 and polydispersity 1.23] was obtained from Sigma Aldrich. Two commercial immobilized lipases, viz Lipolase and Novozyme, were obtained from Novo Nordisk (Denmark) as a gift. Candida Rugosa (activity of 724 units/mg) and hog pancreas 
(activity of 147 units/mg) are from Sigma Aldrich. All other chemicals, such as benzene, chloroform, 1,1,2,2 tetrachloroethane, and tetrahydrofuran were obtained from S. D. Fines (India) and distilled and filtered before use.

\section{Degradation experiments}

A bulk solution of $6 \mathrm{~kg} / \mathrm{m}^{3}$ polycarbonate was prepared in the required solvent. Five batches of $10 \mathrm{~mL}$ each of this solution with $0.01 \mathrm{~g}$ of each lipase were taken in screw cap culture tubes for each temperature study. The water baths were maintained at various temperatures within $\pm 0.1^{\circ} \mathrm{C}$ with proportional-integral derivative (PID) temperature controllers. Samples were constantly stirred to ensure uniform polymer concentration. Aliquots $(0.2 \mathrm{~mL})$ were taken at regular intervals for analysis by gel permeation chromatography (GPC). The enzyme was removed from the sample by centrifugation before analysis. Control experiments were carried out in the absence of enzyme and no degradation was observed. Many experiments were repeated three times and the standard deviation in rate coefficients was less than $2 \%$.

\section{GPC analysis}

The GPC system (Waters) consists of an isocratic pump, a sample loop $(50 \mu \mathrm{L})$, three GPC columns (HR-4, HR-3, and HR-0.5) of varying pore sizes, and an online differential refractive index detector. Tetrahydrofuran was used as eluent at a constant flow rate of $1.0 \mathrm{~mL} / \mathrm{min}$ through the system. Polystyrene standards were used as standards for calibration. The calibration plot and other details are available elsewhere. $^{12}$

\section{Theoretical model}

Lipase degrades polycarbonate by specific chain-end scission producing oligomers of average molecular weight $x_{s}$. Consider the specific chain end scission by the following reaction, with the rate coefficient of $k_{s}$,

$$
P(x) \stackrel{k_{s}}{\rightarrow} P\left(x-x_{s}\right)+Q\left(x_{s}\right)
$$

Here, $P(x)$ represents the polymer of molecular weight $x . Q\left(x_{s}\right)$ represents the specific product of MW, $x_{s}$ and $p(x, t)$ and $q_{t}(t)$ represents concentrations of the polymer and the specific product at any time, respectively. The degradation rate is assumed to directly depend on the polymer concentration and enzyme activity. Assuming $x$ to be a continuous variable, the population balance for the polymer and specific product is

$$
\frac{\partial p(x, t)}{\partial t}=k_{\mathrm{s}} a(t) \int_{x}^{\infty} p\left(x^{\prime}, t\right) \delta\left(x,\left(x^{\prime}-x_{s}\right)\right) d x^{\prime}-k_{\mathrm{s}} a(t) p(x, t)
$$

$$
\frac{\partial q(t)}{\partial t}=\int_{x_{s}}^{\infty} k_{s} a(t) p\left(x^{\prime}, t\right) \Omega\left(x_{s}, x^{\prime}\right) d x^{\prime}
$$

Where $a(t)$ is the activity of the lipase. For the specific chain end scission, the stoichiometric kernel $\Omega\left(x_{s}, x^{\prime}\right)$, which determines the product distribution, is given by $\delta\left(x_{s}, x^{\prime}\right){ }^{13} k_{s}$ is assumed to be independent of the molecular weight as it undergoes specific chain end scission. ${ }^{13}$ Applying moment, $\int_{0}^{\infty} x[f(x)] d x$, on eqs 1 and 2 yields

$$
\begin{gathered}
\frac{d p^{(j)}}{d t}=k_{s} a(t) \sum_{i=0}^{j}\left(\begin{array}{l}
j \\
i
\end{array}\right)\left(-x_{s}\right)^{j} p^{(j-i)}(x, t)-k_{s} a(t) p^{(j)}(x, t) \\
\frac{d q^{(j)}(t)}{d t}=k_{s} a(t) p^{(0)} x_{s}^{j}
\end{gathered}
$$

where ${ }_{i}^{j}$ is the binomial expression. Equation 4 shows the time variation of the specific product moments. The zeroth and first moment represent the molar and mass concentration of the specific product and are obtained by setting $j=0$ and 1 in eqs 3 and 4 . The molar and mass concentration of the polymer and specific products are given by

$$
\begin{gathered}
\frac{d p^{(0)}}{d t}=0 \text { and } \frac{d p^{(1)}}{d t}=-k_{s} a(t) x_{s} p^{(0)} \\
\frac{d q^{(0)}}{d t}=k_{s} a(t) p^{(0)} \text { and } \frac{d q^{(1)}(t)}{d t}=k_{s} a(t) x_{s} p^{(0)}
\end{gathered}
$$

By the molar conservation, the total moles of the polymer $p^{(0)}$ remains constant with time[as shown by eq 5], i. e., $p^{(0)}=p_{0}{ }^{(0)}$. A simplified exponential model is assumed to account for the deactivation of the lipase, $a(t)=a_{0} \exp \left(-k_{d} t\right)$, where $a_{o}$ is the initial activity of the lipase and $k_{d}$ is the enzyme deactivation rate, eq 3 reduces to

$$
\frac{d q^{(1)}(t)}{d t}=k_{s} a_{0} \exp \left(-k_{d} t\right) x_{s} p^{(0)}
$$

Solving Eq 7 with the initial condition $q^{(1)}(t=0)=0$, yields,

$$
\frac{q^{(1)}(t)}{p_{0}^{(1)}}=\frac{k_{s} a_{o} x_{s}}{k_{d} M_{n 0}}\left(1-\exp \left(-k_{d} t\right)\right)
$$




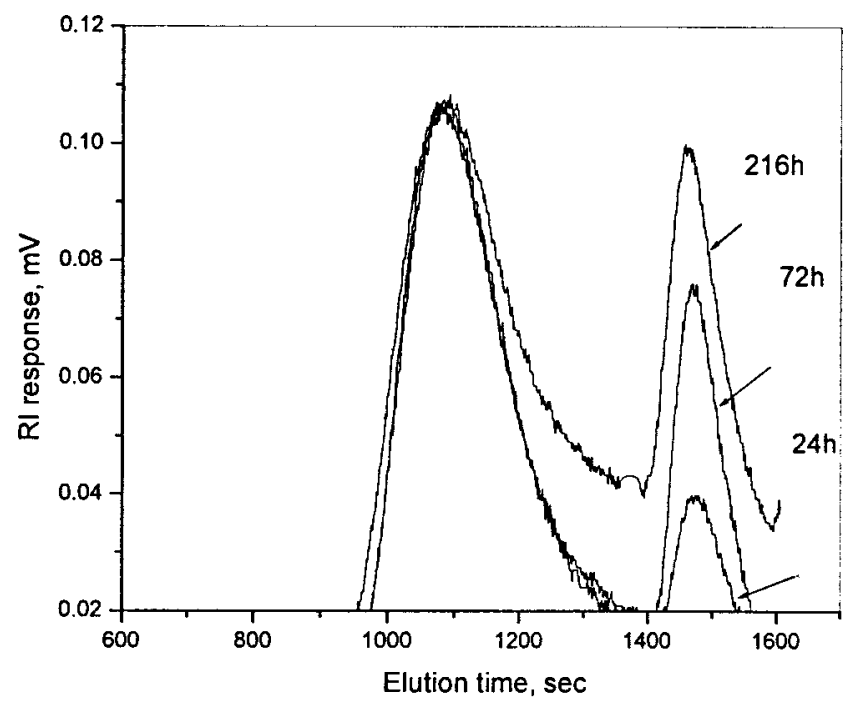

Figure 1 Molecular weight distribution dynamics for the biodegradation of poly (bisphenol-A carbonate) in benzene by lipolase at $55^{\circ} \mathrm{C}$. The numbers on the figure indicate reaction time in hours.

As $t \rightarrow \infty$, eq 8 becomes

$$
\frac{q_{s}}{p_{0}^{(1)}}=\frac{q^{(1)}(t \rightarrow \infty)}{p_{0}^{(1)}}=\frac{k_{s} a_{0} x_{s}}{k_{d} M_{n 0}}
$$

The rationalized mass fraction of specific product formed can be obtained, by omitting the moment indicator, from eqs 8 and 9.

$$
q_{r}(t)=\frac{q^{(1)}(t)}{q^{(1)}(t \rightarrow \infty)}=1-\exp \left(-k_{d} t\right)
$$

$q_{s}$ represents the mass of specific products at infinite time $\left(q^{(1)}(t \rightarrow \infty)\right)$. The $q_{s}$ value used in the above equation was obtained from the experiments after 20 days where the rate of degradation is negligibly small. Equation 10 indicates that a semilogarithmic plot of 1 $-q_{r}(t)$ with time will be straight with the slope $k_{d}$.

\section{RESULTS AND DISCUSSION}

The effect of different lipases on the biodegradation of polycarbonate was investigated in solution at various temperatures. A typical biodegradation profile of polycarbonate (as shown in Fig. 1) by the lipases showed two peaks in the gel permeation chromatograph. The first peak represent the polymer peak at an average MW of 64,000 and the second peak represents a specific product peak at an average MW of 1400 . Before the reaction, there was no specific product peak and as the reaction time increases, the specific product peak increases. As the number average molecular weight of the specific product formed is reasonably constant, the mass fraction of the specific product formed can better enunciate degradation dynamics rather than using the molecular weight. The terms, $q^{(1)}(t)$ and $p_{0}^{(1)}$, represent the mass fraction of the specific products obtained from the area under the specific product peak in the chromatograph and the initial polymer mass, respectively. Thus the quantity, $q^{(1)}(t) / p_{0}^{(1)}$, represents the mass fraction of the specific product to the polymer mass.

It has been observed that the specific oligomeric molecular weight reasonably remained constant around $\sim 1400$ irrespective of type of lipase used and temperature of operation. Thus the value of $x_{s}$ used in the model is 1400 and the initial activity, $\mathrm{a}_{o}$, is assumed to be unity.

Figures 2a-2d depict the degradation profiles for Candida Rugosa, Hog Pancreas, Lipolase and Novozyme 435 respectively. The solid lines in these plots are theoretical fits based on equation 8 , indicating the theory is satisfactory. The deactivation coefficient was determined from the slope of the plot between $\ln (1-$ $\mathrm{q}_{r}(\mathrm{t})$ ) against time as indicated by equation 10 . The specific chain end scission coefficient of degradation was obtained using the equation 9 . The insets in these plots show the semilogarithmic plot as suggested by the equation 10 . These insets again show the validity of the model derived. The ability of the lipases to degrade the polymer was in the order of LL $>C R>N V>H P$. The scission rate coefficient $\left(k_{s}\right)$, enzyme deactivation constant $\left(\mathrm{k}_{d}\right)$ are listed in Table I. The spent polymer showed degradation similar to one observed when fresh batch of enzyme was added indicating that the saturation in the degradation is due to enzyme deactivation.

The effect of temperature on the enzymatic degradation of PC was investigated. At low temperatures, the enzyme was low active while high temperatures are detrimental to the enzyme. Thus an optimal operating temperature for the degradation of PC was determined for various lipases. Figure 3 shows the optimal temperature plot for various lipases. The optimal temperature for degradation of $\mathrm{PC}$ was $50^{\circ} \mathrm{C}$ by $\mathrm{Hog}$ Pancreas lipase whereas and $60^{\circ} \mathrm{C}$ for others.

The effect of solvents on the enzymatic degradation of PC was investigated in various solvents such as benzene, chloroform, tetrahydrofuran, and tetrachloroethane. Though the optimal temperatures for the degradation of $\mathrm{PC}$ by lipolase was $60^{\circ} \mathrm{C}, 55^{\circ} \mathrm{C}$ was chosen to study the effect of solvent, as the boiling point of chloroform and tetrahydrofuran is close to $60^{\circ} \mathrm{C}$. Figure 4 shows the variation of the mass of specific products with time for the degradation of PC by $\mathrm{LL}$ at $55^{\circ} \mathrm{C}$, for various solvents such as benzene, chloroform, tetrahydrofuran and tetrachloroethane. The inset shows the semilogarithmic fit as suggested by the equation 10 . The scission rate coefficients and deactivation coefficients for various solvents are listed 

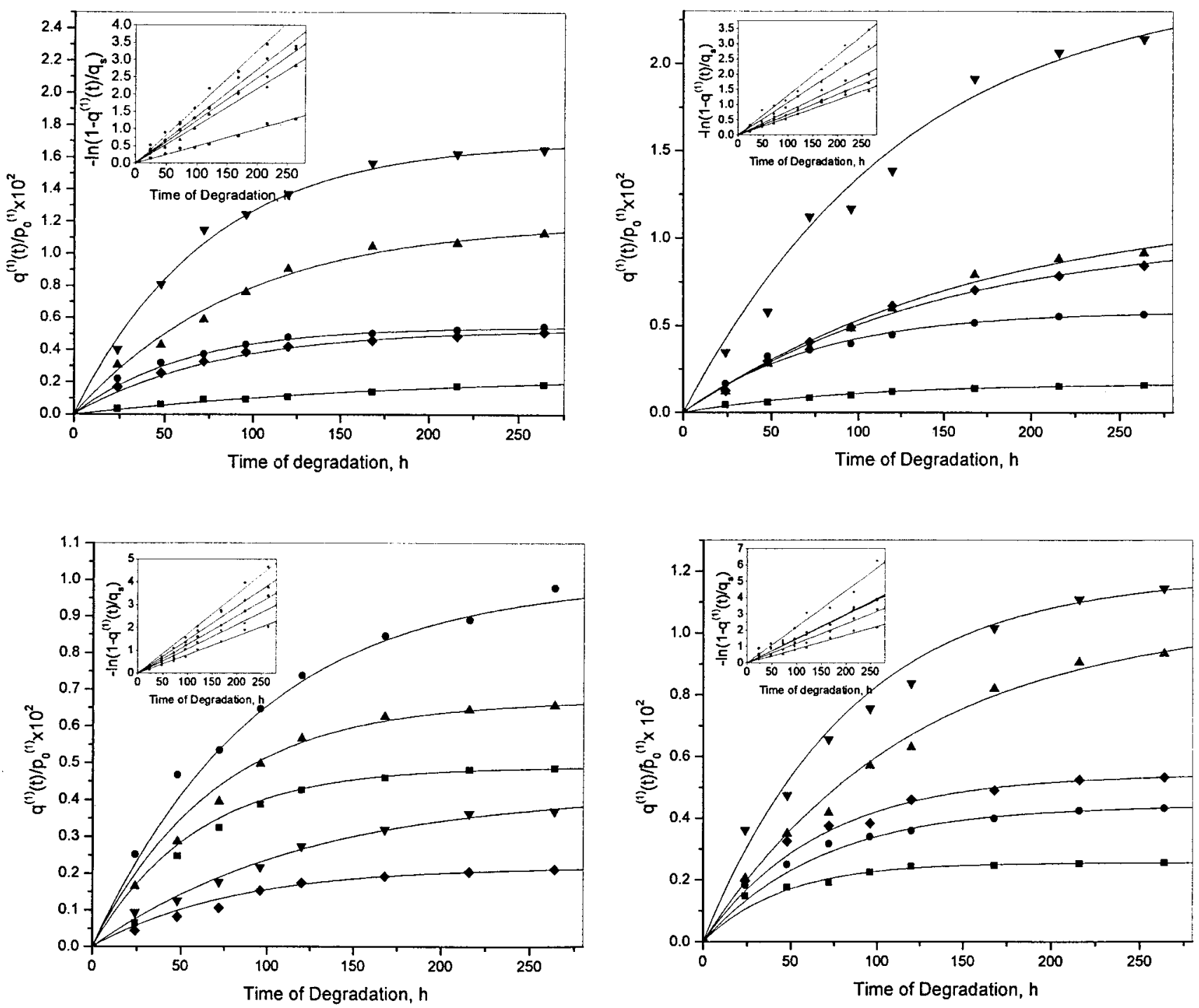

Figure 2 Variation of the mass fraction of the specific product with time in benzene catalyzed by a) Candida Rugosa, b) Hog Pancreas, c) Lipolase, d) Novozyme 435 . Legends: $\mathbf{\square}, 26^{\circ} \mathrm{C} ; \mathbf{0}, 50^{\circ} \mathrm{C} ; \boldsymbol{\Delta}, 55^{\circ} \mathrm{C} ; \boldsymbol{\nabla}, 60^{\circ} \mathrm{C} ; \bullet, 70^{\circ} \mathrm{C}$, lines represent the fit by theory.

in Table II. In the present investigation, it is shown that poly (bisphenol A carbonate) can be degraded in organic media and is affected by the solvent properties. The observed trend in the enzymatic degradation of the polycarbonate cannot be only attrib- uted to the activity of the lipase itself and depends on the type of bonds to be broken and the reaction media. For the same set of enzymes, the trend observed for the degradation of poly (vinyl acetate) ${ }^{14}$ was $\mathrm{HP}>\mathrm{NV}>\mathrm{LL}>\mathrm{CR}$. The solvent properties, such

TABLE I

Rate Coefficients $\left(k_{d} \times 10^{2} \mathrm{~h}^{-1}\right)$ for Polymer Scission and Enzyme Deactivation $\left(k_{s} \times\right.$ $10^{3} \mathrm{~h}^{-1}$ ) for the Degradation of Polycarbonate by Various Lipases in Benzene

\begin{tabular}{|c|c|c|c|c|c|c|c|c|}
\hline \multirow{2}{*}{$\begin{array}{l}\text { Temperature } \\
\left({ }^{\circ} \mathrm{C}\right)\end{array}$} & \multicolumn{2}{|c|}{ CR } & \multicolumn{2}{|c|}{ HP } & \multicolumn{2}{|c|}{ LL } & \multicolumn{2}{|c|}{ NV } \\
\hline & $\mathrm{k}_{\mathrm{d}}$ & $\mathrm{k}_{\mathrm{s}}$ & $\mathrm{k}_{\mathrm{d}}$ & $\mathrm{k}_{\mathrm{s}}$ & $\mathrm{k}_{\mathrm{d}}$ & $\mathrm{k}_{\mathrm{s}}$ & $\mathrm{k}_{\mathrm{d}}$ & $\mathrm{k}_{\mathrm{s}}$ \\
\hline 26 & 0.50 & 0.58 & 1.73 & 3.87 & 1.06 & 0.81 & 2.20 & 2.56 \\
\hline 50 & 1.63 & 4.03 & 1.06 & 4.86 & 1.30 & 3.47 & 1.46 & 2.95 \\
\hline 55 & 1.08 & 5.90 & 1.46 & 4.48 & 0.58 & 3.19 & 0.84 & 4.05 \\
\hline 60 & 1.36 & 10.57 & 0.81 & 1.58 & 0.78 & 8.86 & 1.19 & 6.47 \\
\hline 70 & 1.24 & 2.98 & 1.25 & 1.25 & 0.97 & 3.16 & 1.49 & 3.70 \\
\hline
\end{tabular}




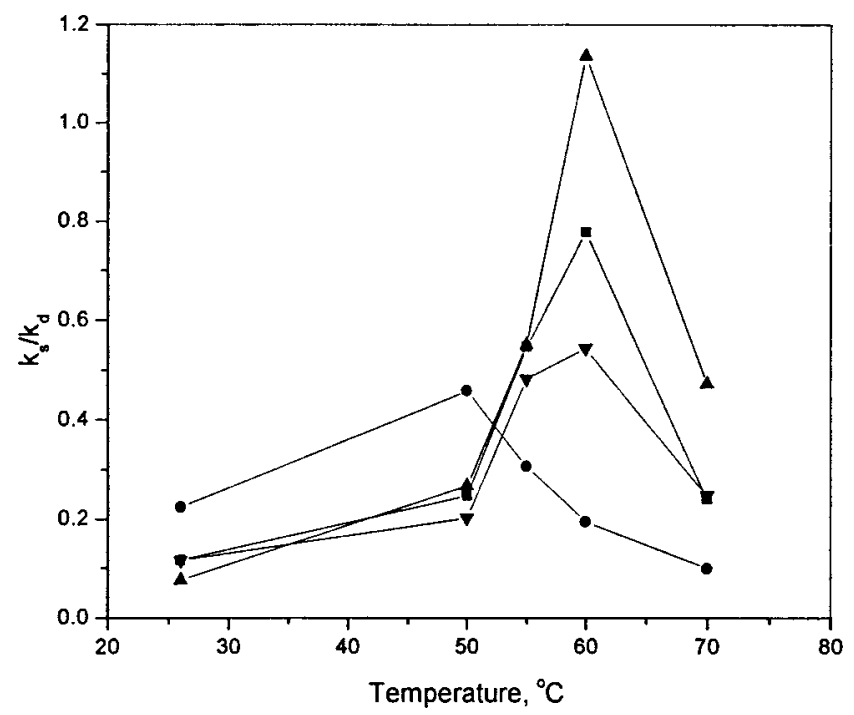

Figure 3 Effect of temperature on the degradation rate coefficient for various lipases. Legends: $\mathbf{\square}$, Candida Rugosa; ๑, Hog Pancreas; $\boldsymbol{\Delta}$, Lipolase; $\boldsymbol{\nabla}$, Novozyme 435

as viscosity and polarity, will affect the degradation of the polymers. While viscosity influences the transport of reactants and enzymes, the polarity influences the enzyme action directly.

Effect of viscosity. Figure 5 shows the effect of viscosity on the degradation of PC by various solvents. The overall composite rate coefficient can be defined as, $\mathrm{ks} / \mathrm{k}_{d}$ was used to represent the degradation behavior. The degradation rate decreased drastically with increase in viscosity. As the viscosity is increased, the fluid transport properties will be reduced resulting in

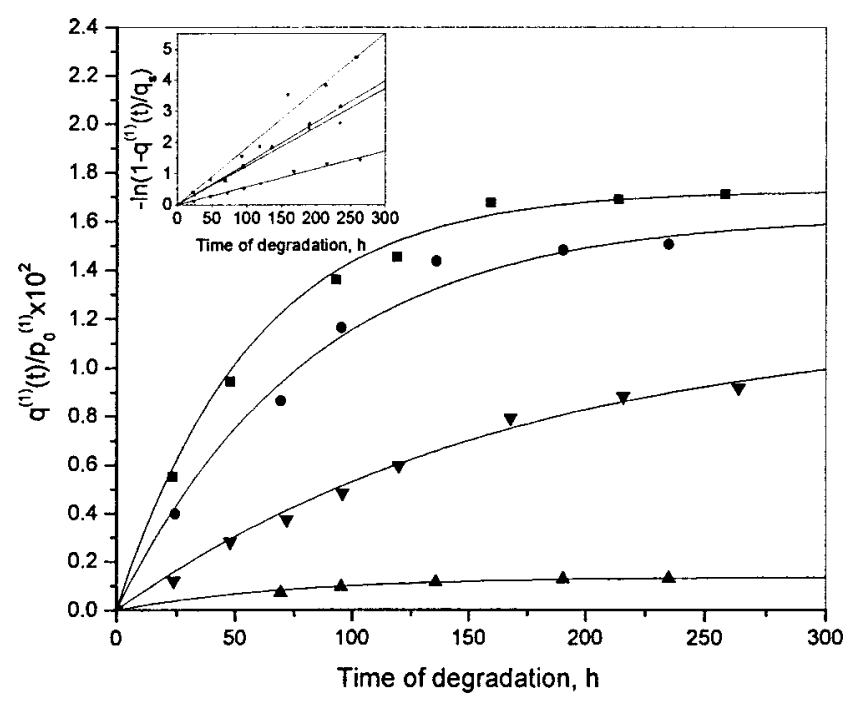

Figure 4 Variation of the mass fraction of specific products with time, catalyzed by $\mathrm{LL}$ at $55^{\circ} \mathrm{C}$, in various solvents. The lines represent the fit by theory. Legends: $\boldsymbol{\Delta}$, tetrachloroethane; $\mathbf{\square}$, tetrahydrofuran; $\boldsymbol{0}$, chloroform; $\boldsymbol{\nabla}$, benzene
TABLE II

Rate Coefficients for Polymer Scission and Enzyme Deactivation Constant for the Degradation of Polycarbonate by Various Lipases at $55^{\circ} \mathrm{C}$ in Different Solvents

\begin{tabular}{lcc}
\hline \multicolumn{1}{c}{ Solvent } & $k_{d} \times 10^{2}, \mathrm{~h}^{-1}$ & $k_{s} \times 10^{3}, \mathrm{~h}^{-1}$ \\
\hline Benzene & 0.58 & 3.19 \\
Chloroform & 1.24 & 9.214 \\
Tetrahydrofuran & 1.78 & 14.04 \\
Tetrachloroethane & 1.32 & 0.818 \\
\hline
\end{tabular}

low facilitated transport of the polymer to enzyme resulting in reduced degradation.

Effect of polarity. The effect of polarity on the degradation rate coefficient was reverse of viscosity. Solvents of lower polarity (i.e., lower hydrophilicity) are less able to disrupt the structure of the necessary tightly bound polymer molecules and thus the degradation rate decreases with decrease in polarity. ${ }^{15}$ The increase in the polarity of the solvent increased the degradation rate except in the case of tetrachloroethane. This indicates that the effect of viscosity is more dominant than the polarity. A similar behavior is observed for the degradation of poly ( $\epsilon$-caprolactone) in various solvents. ${ }^{10}$

\section{CONCLUSION}

The degradation dynamics of poly (bisphenol-A carbonate) by various lipases at various temperatures were determined. The optimal temperatures of each enzyme were determined. The order of increased biodegradability is $\mathrm{LL}>\mathrm{CR}>\mathrm{NV}>\mathrm{HP}$. The increase in viscosity of the solvent decreased the degradation rate while the in-

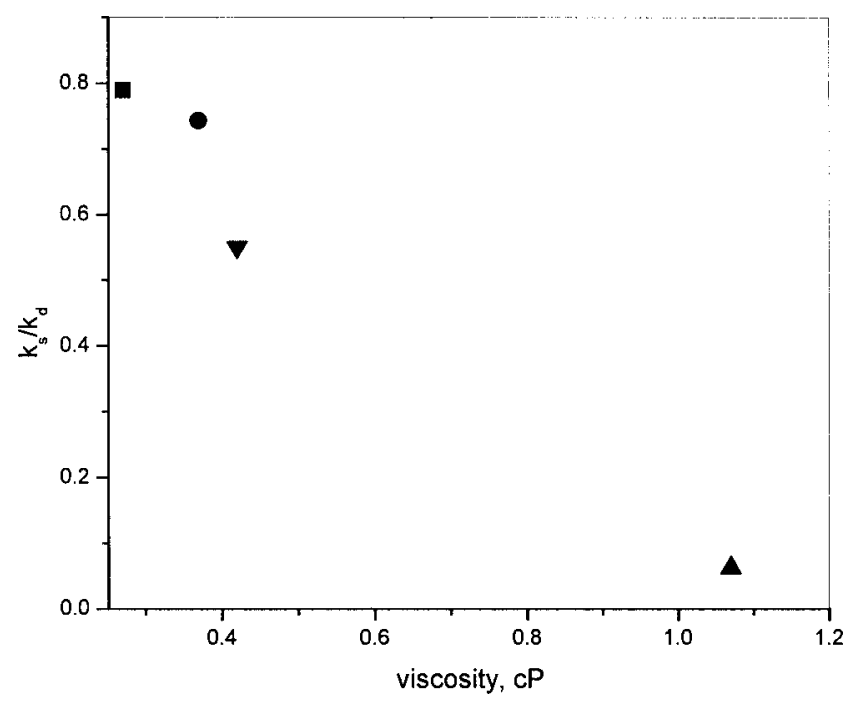

Figure 5 Effect of Viscosity on the degradation of PC by lipolase at $55^{\circ} \mathrm{C}$. See Figure 3 for legend. 
crease in polarity enhanced the degradation behavior. The overall degradation rate is dependent on the controlling limiting resistance due to viscosity and polarity as they are in series. Continuous distribution kinetics model was proposed to explain the degradation dynamics by considering the enzyme deactivation. The rate coefficients were determined which in turn determined the optimal operating point of the degradation.

The authors thank the department of science and technology, India, for financial support.

\section{References}

1. T. Hartmann, Meyer, H. H., Scheper, T. Enzyme and Microbial Technol 2001, 28, 653.
2. B. Combou, Klibanov, A. M. J Am Chem Soc, 1984, 106, 2687.

3. M. Waks. Proteins: Struct, Funct, Genet 1986, 1, 4.

4. D. A. Abramowicz, Keese, C. R. Biotechnol Bioeng 1989, 33, 149.

5. Y. Tokiwa, Suzuki, T. Nature, 1977, 270, 76.

6. Y. Tokiwa, Suzuki, T. J Appl Polym Sci 1981, 26, 441.

7. H. Nishida, Tokiwa, Y. J Environ Polym Degrad 1993, 1, 227.

8. H. Nishida, Tokiwa, Y. Chem Lett 1994, 421.

9. T. Suyama, Tokiwa, Y. Enzyme Microbial Technol 1997, 20, 122.

10. G. Sivalingam, Chattopadhyay, S., Madras, G. Polym Degrad Stab 2003, 79, 413.

11. A. Zaks, Klibanov. Science 1970, 227, 680.

12. G. Sivalingam, Madras, G. Polym Degrad Stab 2003, 80, 11.

13. Y. Kodera, McCoy, B. J. AIChE J 1997, 43, 3205.

14. S. Chattopadhyay, Sivalingam, G., Madras, G. Polym Degrad Stab 2003, 80, 477.

15. M. Matsumoto, Kida K, Kondo K. J Chem Technol Biotechnol 2001, 76, 1070 . 